\title{
Method to enlarge the hologram viewing window using a mirror module
}

\author{
Hoonjong Kang \\ Bilkent University \\ Department of Electrical and Electronics \\ Engineering \\ Bilkent Ankara, 06800 \\ Turkey \\ and \\ Nihon University \\ Department of Electronics and Computer Science \\ 7-24-1 Narashinodai \\ Funabashi-shi, Chiba 2748501 \\ Japan \\ E-mail: hoonjongkang@ hotmail.com
}

Naoyuki Ohmura
Takeshi Yamaguchi
Hiroshi Yoshikawa
Nihon University
Department of Electronics and Computer Science
7-24-1 Narashinodai
Funabashi-shi, Chiba 2748501
Japan

\author{
Seung-Cheol Kim \\ Eun-Soo Kim \\ Kwangwoon University \\ Department of Electronic Engineering \\ 3D Display Research Center \\ 447-1 Wolgye-Dong, Nowon-Gu \\ Seoul 139-701 \\ Korea
}

\begin{abstract}
A liquid crystal panel for a video projector is often used for holographic television. However, its pixel size and pixel number are not enough for practical holographic 3-D display. Therefore, a multipanel configuration is generally used to increase the viewing window and displayed image size, and many spatial light modulators should be used in them. We propose a novel method to increase the viewing window of a holographic display system. The proposed method, which is implemented by using a mirror module and $4-f$ lens set, is to reconfigure the beam shape reflected by a spatial light modulator. The equipment is applied to a holographic display system, which has only a single spatial light modulator; a hologram could be displayed in a wider viewing window by the equipment than that of the conventional method. By the proposed method, the resolution of the reconfigured spatial light modulator has double resolution in the horizontal direction. Inversely, the vertical resolution is decreased. Even if the vertical resolution is decreased, a viewer could get 3-D effect because humans get more 3-D information in the horizontal direction. We have experimented using a liquid crystal on silicon (LcOS), whose resolution is $4096 \times 2160$ pixels. The reconfigured resolution by the mirror module is $8192 \times 1080$ pixels. From the experiments, the horizontal viewing window is almost two times wider than that without the mirror module. As a result, the hologram can be observed binocularly. (๑) 2009 Society of Photo-Optical Instrumentation Engineers. [DOI: $10.1117 / 1.3180869]$
\end{abstract}

Subject terms: viewing window; Fresnel hologram; holographic display; mirror module; 4 - $f$ lens system.

Paper 080974R received Dec. 16, 2008; revised manuscript received May 14 , 2009; accepted for publication May 22, 2009; published online Jul. 30, 2009. This paper is a revision of a paper presented at the SPIE conference on Practical Holography XXII: Materials and Applications, January 2008, San Jose, California. The paper presented there appears (unrefereed) in SPIE Proceedings Vol. 6912.

\section{Introduction}

The real-time holographic video display system (HoloVideo) developed at MIT $^{1}$ can display tens of holographic images in a second. The Holo-Video uses an acousto-optic modulator (AOM) or liquid crystal display (LCD) as the spatial light modulator (SLM). The AOM method was proposed by Professor Benton's group at MIT Media Laboratory. ${ }^{1}$ This method sacrifices the vertical parallax to realize high-speed computation. In order to make the propagating fringes in the crystal appear stationary, it needs to use mechanical scanning. On the other hand, the optical system with an LCD does not require such mechanical scanning. ${ }^{2}$ How to increase the hologram viewing window is one of the major issues in the research field of holographic television, because the viewing window is usually dependent on the SLM. There are several ways to solve this problem using multichannels of LCD or AOM. ${ }^{3,4}$ Recently, a method to increase the horizontal viewing window using a single SLM has been reported. ${ }^{5-7}$

In this paper, we propose a novel method to increase the

0091-3286/2009/\$25.00 @ 2009 SPIE viewing window of a holographic display system. The proposed method, which is implemented by using a mirror module and 4- $f$ lens set, is to reconfigure the beam shape reflected by a spatial light modulator. The equipment is applied to a holographic display system, which has only a single spatial light modulator; a hologram could be displayed in a wider viewing window by the equipment than that of the conventional method. By the proposed method, the resolution of the reconfigured spatial light modulator has double resolution in the horizontal direction. Inversely, the vertical resolution is decreased. Even if the vertical resolution is decreased, a viewer could get 3-D effect because humans get more 3-D information in the horizontal direction. We have experimented using a liquid crystal on silicon $(\mathrm{LCOS})$, whose resolution is $4096 \times 2160$ pixels. The reconfigured resolution by the mirror module is 8192 $\times 1080$ pixels. From the experiment, the horizontal viewing window is almost two times wider than that of the conventional method without the mirror module. As a result, the hologram can be observed binocularly.

This paper is organized as follows: Section 2 discusses the Fresnel hologram. The proposed method and the designed equipment are discussed in Sec. 3. The analyzed 


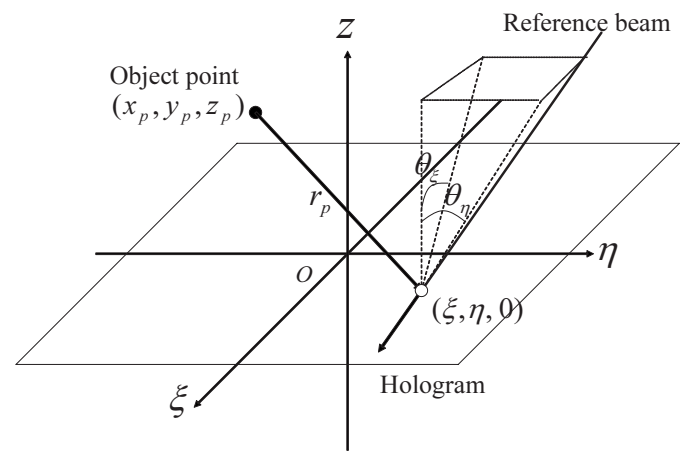

Fig. 1 Model to calculate the Fresnel hologram.

data as to the proposed method are described in Sec. 4. The system configuration of the proposed method and its results are presented in Sec. 5. Last, we conclude this paper in Sec. 6.

\section{Computer-Generated Fresnel Hologram}

In this section, we describe some theory to calculate the Fresnel hologram in order to apply it to the proposed wide holographic display system. First, a rigorous calculation method is described, based on an exact optical model. The object to be recorded is approximated as a collection of self-illuminated points and located at certain point in a system of Cartesian coordinates. The calculation geometry of the hologram is shown in Fig. 1. The hologram is located on the $x-y$ plane. The location of the $p$ 'th point of a 3-D object is specified as $\left(x_{p}, y_{p}, z_{p}\right)$. Each point has real-valued amplitude $a_{p}$ and relative phase $\phi_{p}$. The complex amplitude $O(\xi, \eta)$ on the hologram is determined from the superposition of the object wavefronts by

$O(\xi, \eta)=\sum_{p=1}^{N} \frac{a_{p}}{r_{p}} \exp \left[j\left(k r_{p}+\phi_{p}\right)\right]$,

where $N$ is the number of object points. The wave number $k$ is defined as $k=2 \pi / \lambda$, where $\lambda$ is the free-space wavelength of the light. Note that the factor $\exp (j \omega t)$ is not included explicitly. The oblique distance $r_{p}$ between the $p$ 'th object point and the point $(\xi, \eta)$ on the hologram is defined as

$r_{p}=\left[\left(\xi-x_{p}\right)^{2}+\left(\eta-y_{p}\right)^{2}+z_{p}^{2}\right]^{1 / 2}$.

If the reference beam is collimated, the complex amplitude of the reference beam $R(\xi, \eta)$ is represented as

$R(\xi, \eta)=a_{R} \exp \left[-j k\left(\xi \sin \theta_{\xi}+\eta \sin \theta_{\eta}\right)\right]$,

where $a_{R}$ is real-valued amplitude, $\theta_{\xi}$ is the incident angle of the reference beam on the $\xi$ axis, and $\theta_{\eta}$ is the incident angle of the reference beam on the $\eta$ axis. The total complex amplitude on the hologram plane is the interference of

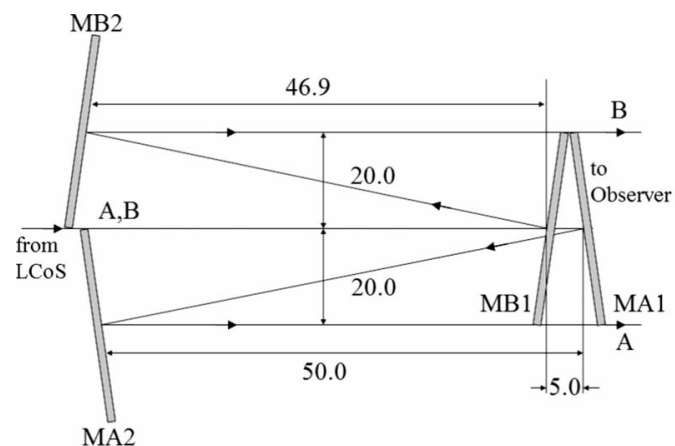

(a)

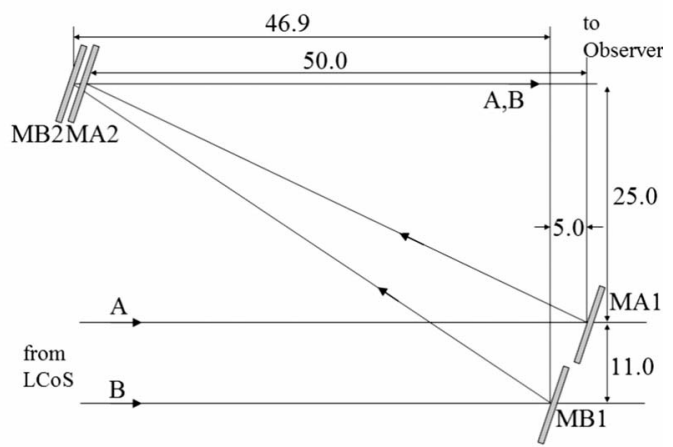

(b)

Fig. 2 Schematic of the mirror module to reconfigure the SLM: (a) side view, (b) top view.

the object beam and the reference beam, represented as $O(\xi, \eta)+R(\xi, \eta)$. The total intensity pattern,

$I_{F H}(\xi, \eta)=|O+R|^{2}=|O|^{2}+|R|^{2}+2 \operatorname{Re}\left\{O R^{*}\right\}$,

is a real physical light distribution on the hologram, where $\operatorname{Re}\left\{O R^{*}\right\}$ is the real part of the complex number $O R^{*}$, and $R^{*}$ is the complex conjugate of $R$. The first term represents the object's self-interference, and the second term is the reference beam intensity. The third term is the interference of the object beam and the reference beam and contains the holographic information.

\section{Beam Reconfiguration}

\subsection{Beam Reconfiguration Using Mirror Module}

Recently, high-resolution LCDs with wide aspect ratios have become available. These LCDs can display even more information than previous LCDs. And their pixel pitch is also decreasing rapidly. In order to display a hologram, they are usually utilized as the SLM. However, their pixel pitch is not yet enough for practical holographic display.

In order to increase the viewing window, the beam reconfiguration method was applied by the proposed mirror module. The mirror module could reconfigure the diffracted beam shape by the SLM. The schematic of the mirror module is shown in Fig. 2, and a photograph is shown in Fig. 3. This module consists of four mirrors, of which two mirrors are held in the upper side, and the others are held in the lower side. The diffracted light on the $\mathrm{LCoS}$ is reflected on the lower mirrors, MA1 and MB1, and reflected again on the upper mirrors, MA2 and MB2. In more detail, the lower 


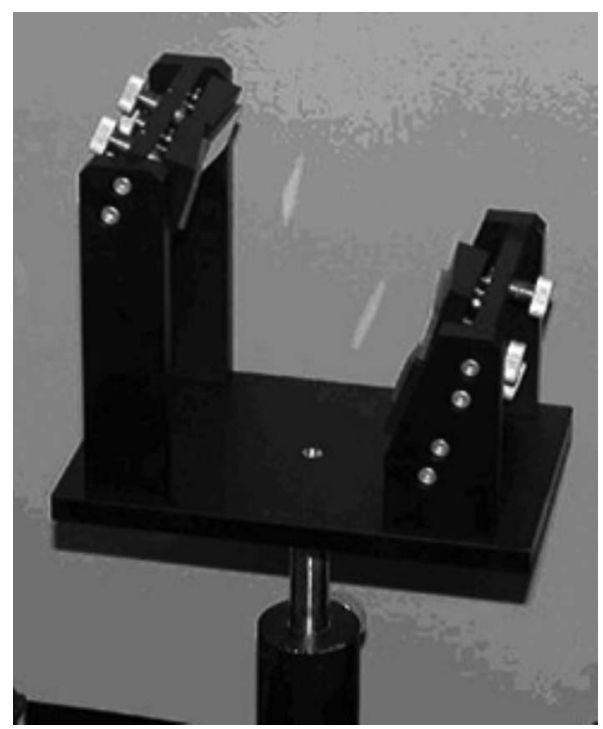

Fig. 3 Photograph of part of the novel optical system.

mirrors operate to divide the resolution in vertical direction, and the upper mirrors operate to rearrange the divided SLM in the horizontal direction to reconfigure the wide SLM in the horizontal direction. For example, the lower mirror MA1 reflected the diffraction light to the upper mirror MA2. On the other hand, MB1 reflected the diffraction light to MB2. Using the mirror module, the resolution of the reconfigured SLM has double resolution in the horizontal direction. Inversely, the vertical resolution is decreased.

\subsection{Pixel Reconfiguration}

The shape of the reconfigured SLM is different from that of the real SLM. Therefore, the computer-generated hologram (CGH) should be generated as to the reconfigured SLM. Then, the generated hologram should be rearranged to match the real SLM. Its conceptual diagram is shown in Fig. 4. At first, the resolution of the used SLM is divided into small vertical segments, and then these are rearranged in the horizontal. The digital hologram for the wide holographic display system is generated on the redefined resolution.. According to this method, it could be made the wide and high resolution SLM in horizontal. Although the resolution is decreased in the vertical direction, the observer can get a 3-D effect from the hologram, because humans get more 3-D information in the horizontal direction.

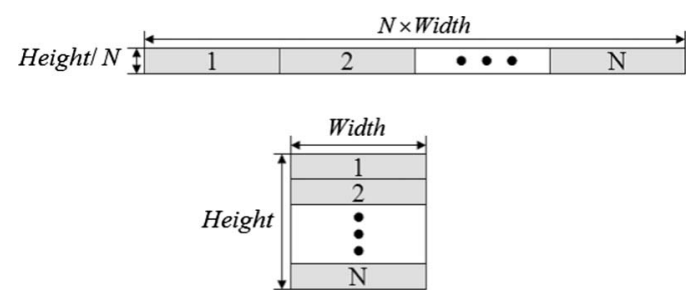

Fig. 4 Optimizing pixel configuration.

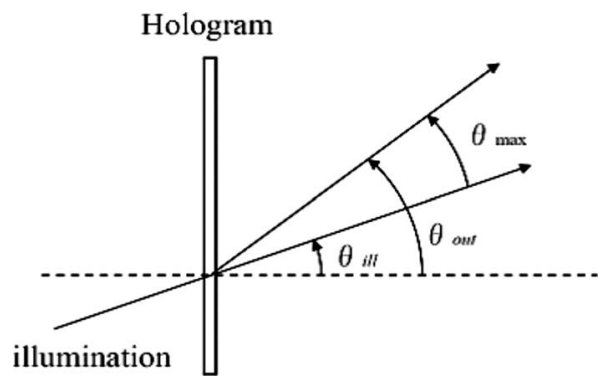

Fig. 5 Model to calculate the diffraction angle.

\section{Benefit of Hologram Viewing Window}

In this section, we consider the efficiencies of the hologram viewing window and diffraction according to the proposed method. In addition, the method was compared with the conventional method, which uses only one SLM.

\subsection{Diffraction Angle}

An LCoS is utilized to display the hologram as an SLM. The maximum diffraction angle of the hologram depends on the pixel pitch of the SLM used. The relation between the output angle, $\theta_{\text {out }}$, and the illumination angle, $\theta_{i l l}$, is shown in Fig. 5, and is determined by ${ }^{8}$

$\frac{\lambda}{2 p}=\sin \theta_{\text {out }}-\sin \theta_{\text {ill }}$,

where $\lambda$ is wavelength of the laser, and $p$ is pixel pitch of the LCoS. The maximum diffraction angle $\theta_{\max }$ is expressed as

$\theta_{\max }=\theta_{\text {out }}-\theta_{\text {ill }}$

and if $\theta_{i l l}=0$,

$\theta_{\text {max }}=\theta_{\text {out }}=\sin ^{-1} \frac{\lambda}{2 p}$.

\subsection{Conventional Method}

As an LCoS is utilized as the SLM in the holographic display system, the hologram viewing window can be expressed as

$V_{h}=W_{h}+2 D \tan \theta_{d}$,

$V_{v}=W_{v}+2 D \tan \theta_{d}$,

where $V_{h}$ and $V_{v}$ are the horizontal and vertical viewing window sizes, respectively; $W_{h}$ and $W_{v}$ are the LCoS sizes in the horizontal and vertical, respectively; $D$ is the distance from the LCoS to viewpoint, and $\theta_{d}$ is the diffraction angle of the LCoS. For example, if the pixel pitch is $9.5 \mu \mathrm{m}$ and the wavelength is $633 \mathrm{~nm}$, the maximum diffraction angle $\theta_{d}$ is $1.90 \mathrm{deg}$. Accordingly, the viewing window at $500 \mathrm{~mm}$ from the LCoS, whose resolution is 4096 $\times 2160$ pixels, is about $72 \mathrm{~mm} \times 54 \mathrm{~mm}$. This viewing window size will be used as the basis of comparison as to the benefit. The simulation environment is shown Table 1. 
Table 1 Simulation environment.

\begin{tabular}{lc}
\hline \hline & \\
SLM resolution & $4096 \times 2160$ pixels \\
Resolution of reconfigured SLM & $8192 \times 1080$ pixels \\
SLM pixel pitch & $9.5 \mu \mathrm{m}$ \\
Light wavelength & $633 \mathrm{~nm}$ \\
Viewing distance & $500 \mathrm{~mm}$ \\
\hline \hline
\end{tabular}

\subsection{With Mirror Module}

The schematic of the SLM reconfiguration method using only the proposed mirror module is shown in Fig. 6. This figure shows how the diffracted light on the SLM is passed by the mirror module and its diffraction characteristics. In this analysis, the distance, $r_{1}$ and $r_{2}$, between the SLM and mirrors is $50 \mathrm{~mm}$, and the viewing distance is $500 \mathrm{~mm}$ from upper mirrors to observer. Therefore, it was assumed that the mirror size is the same as the segment size, which is the SLM divided logically in vertical direction.

First, the diffracted light on the SLM, whose size is $38.9 \mathrm{~mm} \times 20.5 \mathrm{~mm}$, is reflected by the lower mirrors. The size of the diffraction window on the lower mirrors is $42.3 \mathrm{~mm} \times 23.9 \mathrm{~mm}$. However, since the mirror size is smaller than that of the diffraction window, only the part whose size is the same as the SLM is reflected by the lower mirrors. Then, its diffraction benefit is $79.2 \%$.

Second, the light reflected by the lower mirrors is reflected again by the upper mirrors. The diffraction window on the upper mirrors is also $42.3 \mathrm{~mm} \times 23.9 \mathrm{~mm}$, because the light propagates to its diffracted direction. The diffraction benefit as to the upper mirrors is $79.2 \%$. Accordingly, the total diffraction benefit is $62.8 \%$, and the hologram display window to the observer is $111.2 \mathrm{~mm} \times 43.6 \mathrm{~mm}$, an increase of $124.6 \%$. The display window is increased 1.5

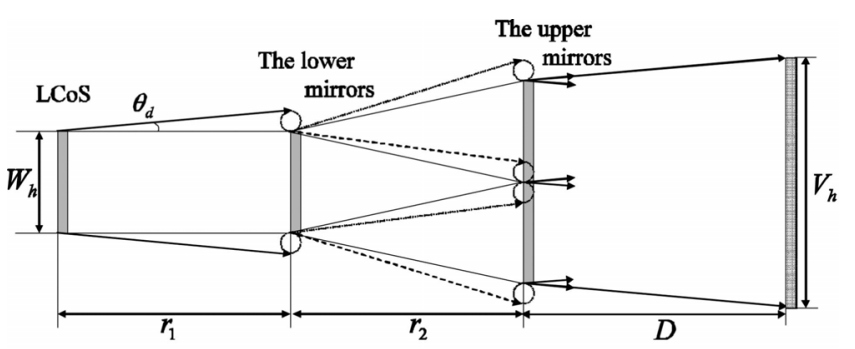

(a)

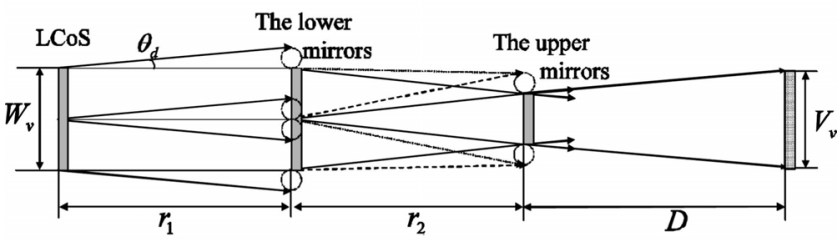

(b)

Fig. 6 Diffracted light on the LCoS and reflected light on the mirror corresponding to the using only mirror model: (a) top view, (b) side view.

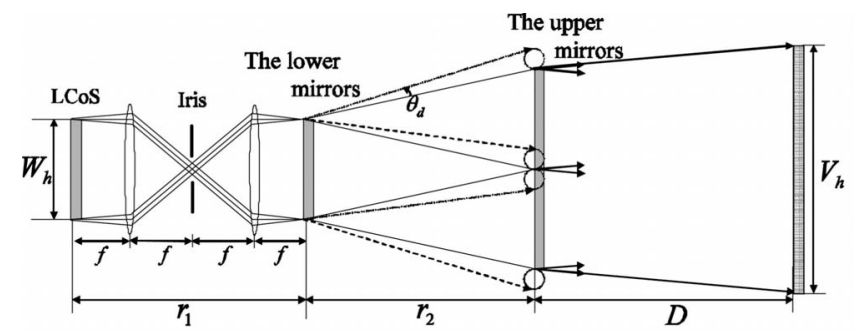

(a)

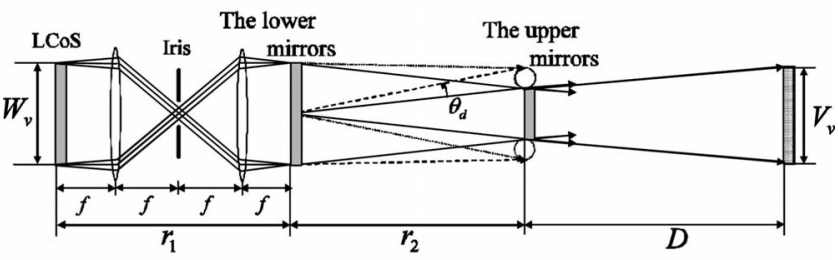

(b)

Fig. 7 Diffracted light on the LCoS and reflected light on the mirror corresponding to the using mirror model and 4-f lens system: (a) top view, (b) side view.

times in the horizontal direction and decreased 0.8 times in the vertical direction. Although the total display window is increased, this method is not suitable to be observed, because the total diffraction benefit is not enough.

\subsection{With Mirror Module and 4-f Lens System}

If the SLM and the mirror module are just used in the display system to reconfigure the diffracted light on the SLM, this method could not have satisfactory diffraction benefit, as mentioned in the previous section. Therefore, a 4- $f$ lens system is involved to improve the diffraction benefit of the proposed method. The schematic as to the mirror module with the 4- $f$ lens set is shown in Fig. 7. The 4- $f$ lens set is placed between the LCoS and the lower mirrors. The diffracted light on the SLM is passed through the two lenses, the 4- $f$ lens set, and they are imaged on the lower mirrors. This means that the SLM is moved at the position of the lower mirrors, and the incident light on each segment on the SLM is diffracted and propagated for each upper mirror. Accordingly, the diffraction benefit could be increased because the loss of diffracted light on the mirrors occurs only on the upper mirrors. The display window by means of this method is $111.2 \mathrm{~mm} \times 43.6 \mathrm{~mm}$. Although this size is the same with the previous method, the diffraction benefit, $79.2 \%$, is increased as result.

A mirror, whose size is larger than the divided segment size of the SLM, can be utilized in the mirror module to cover the whole diffracted light on the SLM. Then, the loss of the diffracted light occurs only on the adjoining part of the upper mirrors. Therefore, the diffraction benefit could be increased up to $96.1 \%$. Its display window is also $111.2 \mathrm{~mm} \times 43.6 \mathrm{~mm}$. The display window and diffraction benefit according to each method are shown in Table 2. As show in Table 2, these could be achieved to display a hologram in a wide observable window using a large mirror module and 4- $f$ lens set. 
Table 2 Characteristics of the optical holographic display system.

\begin{tabular}{lcc}
\hline \hline Display system & Display window $\left(V_{h} \times V_{v}\right)$ & Diffraction benefit \\
\hline Only SLM & $72.3 \mathrm{~mm} \times 53.9 \mathrm{~mm}$ & $100 \%$ \\
With mirror module & $111.2 \mathrm{~mm} \times 43.6 \mathrm{~mm}$ & $62.8 \%$ \\
With mirror module and 4- $f$ lens set & $111.2 \mathrm{~mm} \times 43.6 \mathrm{~mm}$ & $79.2 \%$ \\
With large mirror module and 4- $f$ lens set & $114.5 \mathrm{~mm} \times 46.9 \mathrm{~mm}$ & $96.1 \%$ \\
\hline \hline
\end{tabular}

\subsection{Noise and Interval Effect Due to the Folding Mirror}

\subsubsection{Noise on reconstruction}

In this manuscript, the basic idea is partition of a fringe pattern on SLM optically and reconfiguration of the segmented fringe pattern on a display plane to achieve a wide holographic display. However, the reconstructed image from the reformed fringe pattern by the SLM reconfiguration method with only mirror module has some noise. This noise is caused by the mismatched diffracted light, which is reflected on the opposite mirror. As shown in Fig. 6, some parts of the diffracted light on the lower side and upper side on the SLM are propagated to the opposite mirror in the lower mirror set. In addition, some parts of the reflected light on the lower mirror set are propagated to the opposite mirror in the upper mirror set. Therefore, some diffracted light corresponding to the left side on the display plane is propagated to the right side on the display plane, and some diffracted light corresponding to the right side is propagated to the left side. This mismatched light makes noise on the display plane. This noise is the overlapped reconstruction, which consists of the expected reconstruction and reflected reconstruction from the opposite mirror. This noise can be removed by the $4-f$ lens system. A fringe pattern on the SLM is imaged on the lower mirror set by means of the 4- $f$ lens system. Therefore, the diffracted light from each fringe pattern corresponding to the left side and right side on the display plane is propagated to the accurate direction without any overlapping light. As shown in Fig. 7, the fringe pattern is imaged on the lower mirror set. Even if some part of the reflected light by the lower mirror set is propagated to the opposite mirror in the upper mirror set, as

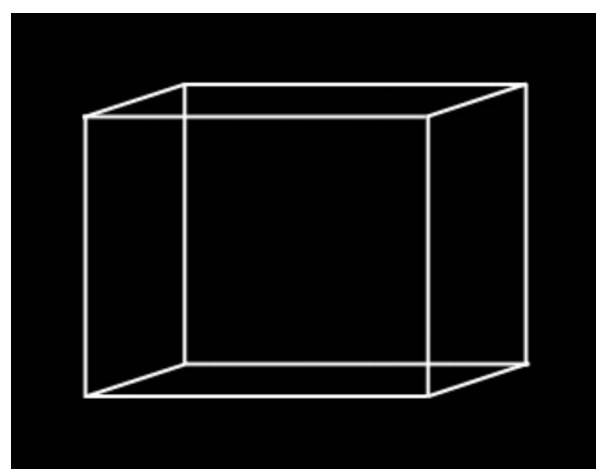

Fig. 8 Perspective image. shown in Fig. 7(a), this reflected light is propagated to outside the available viewing zone. Therefore, the noise, the overlapped image with the left side image and right side image, does not occur in the viewing zone.

\subsubsection{Interval in mirror set}

The mirror module is used in the proposed method. As mentioned earlier, the mirror module consists of four mirrors, two mirrors for the lower mirror set and the other two mirrors for the upper mirror set. In each mirror set, two mirrors are separated and have a narrow interval due to the folding mirror. This interval may cause fringe loss. If this method is utilized for a 2-D image display, image loss will cause a serious problem. However, in the case of the hologram, diffracted light from a whole fringe pattern makes a single reconstruction on a space. On the other hand, the contribution corresponding to a single point is from the whole fringe area. Therefore, even if some information is lost by the interval in the mirror set, the reconstruction does not get any distortion or noise.

\section{Experiment and Result}

In this experiment, an LCoS is used to display the hologram. A cube, shown in Fig. 8, is employed as the model to generate the hologram because it is easy to notice the viewing direction. The generated hologram, calculated by Eq. (1) to Eq. (4), is displayed three ways: using only the SLM, with the mirror module, and with the large mirror module

Table 3 Characteristics of the optical holographic display system.

\begin{tabular}{ccc}
\hline \hline LCoS & Resolution & $4096 \times 2160$ pixels \\
& Pixel pitch & $9.5 \mu \mathrm{m}$ \\
& Refresh rate & $62.5 \mathrm{~Hz}$ \\
& Grayscale level & $8 \mathrm{bits}$ \\
$\begin{array}{c}\text { Optical } \\
\text { setup }\end{array}$ & Wavelength & $633 \mathrm{~nm}$ \\
& Focal length & $300 \mathrm{~mm}$ \\
& of lens set & \\
Viewing & Laser power & $25 \mathrm{~mW}$ \\
distance & & $500 \mathrm{~mm}$ \\
\hline
\end{tabular}




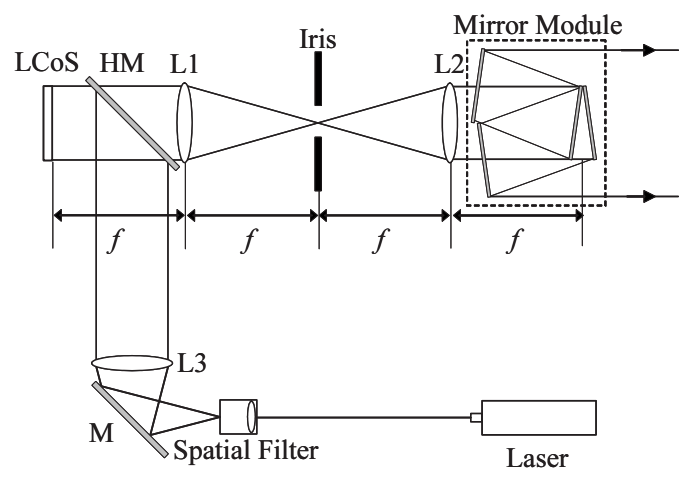

Fig. 9 Schematic of the holographic display system for beam reconfiguration.

including the 4- $f$ lens set. The characteristics of the display system are described in Table 3. The schematic of the optical holographic display system with the mirror module and 4- $f$ lens set is shown in Fig. 9. In Fig. 9, the laser beam is expanded by the spatial filter to match to the shape of the

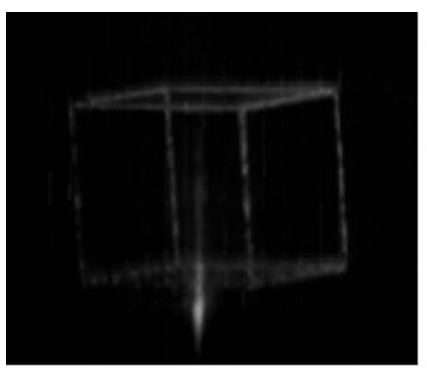

(a)

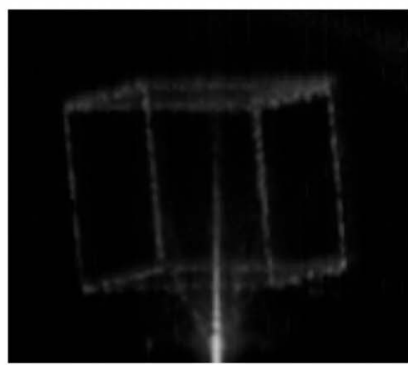

(c)

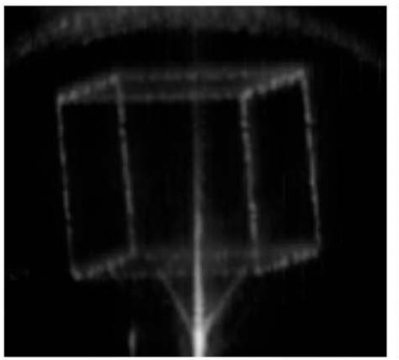

(d)

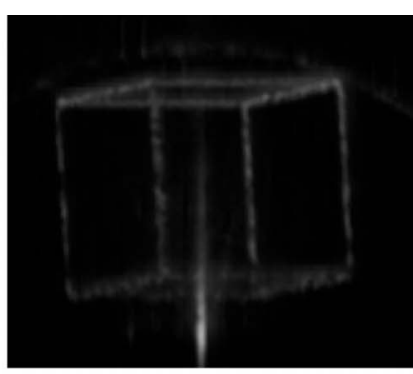

(b)

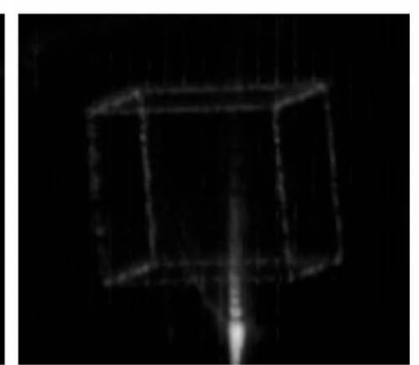

(e)
Fig. 10 Reconstructed images by the optical system with the mirror module and 4- $f$ lens set: (a) $-50 \mathrm{~mm}$ from center, (b) $-25 \mathrm{~mm}$ from center, (c) $0 \mathrm{~mm}$ from center, (d) $25 \mathrm{~mm}$ from center, and (e) $50 \mathrm{~mm}$ from center.
Table 4 Performance of the proposed method.

\author{
Reconfigured resolution \\ Diffraction benefit \\ Display window $\left(V_{h} \times V_{v}\right)$ \\ Diffraction angle \\ Mirror size
}

Viewing distance
$8192 \times 1080$ pixels

$96.1 \%$

$114.5 \mathrm{~mm} \times 46.9 \mathrm{~mm}$

$1.90 \mathrm{deg}$

$43 \mathrm{~mm} \times 14 \mathrm{~mm}$

$500 \mathrm{~mm}$
LCoS. Then, the beam is collimated with the lens, L3. The beam is reflected by the half-silvered mirror and illuminates the LCoS. The incident beam is diffracted on the LCoS and imaged on the lower mirrors of the mirror module through the 4- $f$ lens set. Last, the reconfigured beam by means of the mirror module forms a reconstructed image in the air.

Using the mirror module, the measured width of the viewing window in the SLM reconfiguration method with 4- $f$ lens system is $110 \mathrm{~mm}$, almost the same as the theoretical value of $111.2 \mathrm{~mm}$. Even if the 4- $f$ lens system is not utilized, this method also has the same viewing window width of $110 \mathrm{~mm}$. However, as mentioned in Sec. 4.5, some noise occurs on the display plane. Therefore, by means of the mirror module and the 4- $f$ lens system, a wide viewing window without noise caused by the mismatched diffracted light could be achieved.

The reconstructed images by the optical system with the mirror module and 4- $f$ lens set are shown in Fig. 10. In addition, the performance of the designed method is shown in Table 4. In Fig. 10, one can notice that the proposed method gives a clear reconstructed image in a wide viewing zone. In addition, if the viewpoint is moved from center to the right side or left side, the observer could recognize the motion parallax. Consequently, through the proposed method, an observer could see the displayed holographic image in the wide viewing window and be able to get 3-D effects in the displayed holographic image.

\section{Conclusion}

In this paper, we presented a novel holographic display method to enlarge the viewing window of a hologram viewing zone. A beam reconfiguration method by means of a mirror module has been designed. Additionally, an optical holographic display system was built with the mirror module, and the reconstructed images displayed in wide viewing window were shown. As a result, the designed method showed that a digital hologram can be displayed in a wide viewing window whose size is $114.5 \mathrm{~mm} \times 46.9 \mathrm{~mm}$.

\section{Acknowledgments}

This research was supported by the Ministry of Knowledge Economy (MKE) of Korea under the Information Technology Research Center (ITRC). Support Program supervised by the Institute of Information Technology Assessment (IITA); IITA-2008-C1090-0801-0018. 


\section{References}

1. P. St. Hilaire, S. A. Benton, M. Lucente, M. L. Jepsen, J. Kollin, H. Yoshikawa, and J. Underkoffler, "Electronic display system for computational holography," in Practical Holography IV, S. A. Benton, Ed., Proc. SPIE 1212, 174-182 (1990).

2. F. Mok, J. Diep, H. Liu, and D. Psaltis, "Real-time computer generated hologram by means of liquid-crystal television spatial light modulator," Opt. Lett. 11(11), 748-750 (1986).

3. P. St. Hilaire, S. A. Benton, M. Lucente, J. D. Sutter, and W. J. Plesniak, "Advances in holographic video," in Practical Holography VII, Proc. SPIE 1914, 188-196 (1993).

4. K. Maeno, N. Fukaya, O. Nishikawa, K. Sato, and T. Honda, "Electro-holographic display using 15M pixels LCD," in Practical Holography IX, S. A. Benton, Ed., Proc. SPIE 2652, 15-22 (1996).

5. Y. Hayashi and Y. Takaki, "Horizontal resolution enhanced hologram to increase horizontal viewing angle," in Practical Holography XXII, Proc. SPIE 6912, 69120I (2008).

6. N. Ohmura, H. Kang, T. Yamaguchi, and H. Yoshikawa, "A method, to increase the hologram viewing angle by the beam reconfiguration," in Practical Holography XXII, Proc. SPIE 6912, 691200 (2008).

7. J. Hahn, H. Kim, Y. Lim, G. Park, and B. Lee, "Wide viewing angle dynamic holographic stereogram with a curved array of spatial light modulators," Opt. Express 16(16), 12372-12386 (2008).

8. E. Hecht, Optics, Wesley Longman, New York (2002).

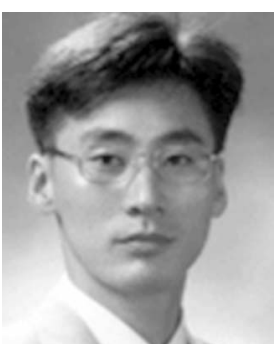

Hoonjong Kang graduated from Kwangwoon University with a bachelor of engineering degree in 1998. He joined the 3D Korea company in 2000 , received a master of engineering degree from Kwangwoon University in 2001, and joined the government institute ETRI in 2002. He received a doctor of engineering degree from Nihon University in 2008 and joined the European FP7 Real3D project in 2008. His research interests include computer-generated holograms, electroholography, 3-D display, and signal processing of stereoscopic images.

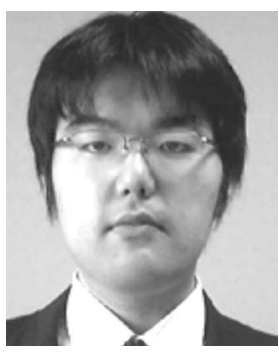

Naoyuki Omura received BS and MS degrees in electrical engineering from Nihon University in 2006 and 2008, respectively. His current research interests are in the areas of electroholography and computergenerated holograms.

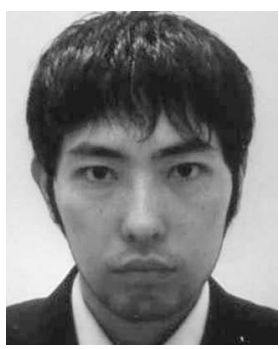

Takeshi Yamaguchi received BS and MS degrees in electronic engineering from $\mathrm{Ni}-$ hon University in 2004 and 2006, respectively. Since 2006, he has been on the faculty at Nihon University. His current research interests are in electroholography and computer-generated holograms.

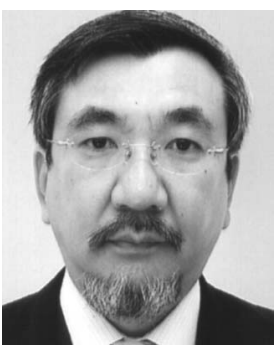

Hiroshi Yoshikawa received a BS degree, an MS degree, and a PhD from Nihon University, all in electrical engineering, in 1981 1983 , and 1985, respectively. He joined the faculty at Nihon University in 1985, where he currently holds the position of professor of electronics and computer science. From 1988 to 1990 , he was a research affiliate of MIT Media Laboratory. His current research interests are in electroholography, computer-generated holograms, display holography, and computer graphics.

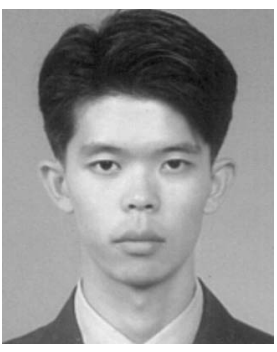

Seung-Cheol Kim received his BS degree from Kwangwoon University, Seoul, Korea, in 2002 and his MS and PhD degrees in electronic engineering from the Graduate School of Kwangwoon University in 2004 and 2007, respectively. Since 2007, he has been a research professor at the 3D Display Research Center of Kwangwoon University. His research interests include $3-D$ imaging and display, holography, and optical information processing.

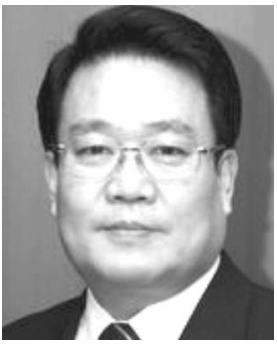

Eun-Soo Kim joined the faculty of Kwangwoon University in 1981, where he is presently a professor at the Department of Electronic Engineering. He was a visiting professor in the Department of Electrical Engineering, CalTech. In 2000, his research laboratory was honored as a National Research Laboratory by the Ministry of Science and Technology of Korea, and his 3DRC (3D Display Research Center) was also honored as an outstanding ITRC (Information Technology Research Center) by the Ministry of Communication and Information of Korea in 2003. He is currently the acting president of the 3D Fusion Industry Consortium and the presidentelect of the Korean Information and Communications Society. He established the International Workshop on 3D Information Technology in 2004, and he co-organized the International 3D Fair in collaboration with the 3D Consortium of Japan in 2006. His research interests include 3-D imaging and displays, 3-D fusion technologies, and their applications. 